\title{
Bipolar Triangular Wave Current Pulse Inverter for Shallow TEM Survey
}

\author{
Zhao Yifu ${ }^{1,2}$, Liu Lihua ${ }^{1, *}$, Geng Zhi ${ }^{1}$, and Liu Xiaojun ${ }^{1}$ \\ ${ }^{1}$ Institute of Electronics, Chinese Academy of Sciences Key Laboratory of Electromagnetic Radiation and Sensing Technology, Chinese \\ Academy of Sciences, Beijing 100190, China \\ ${ }^{2}$ University of Chinese Academy of Sciences School of Electronic, Electrical and Communication Engineering, Beijing 100049, China
}

\begin{abstract}
A bipolar triangular wave current pulse inverter for shallow TEM survey is described. It can generate a peak current more than $10 \mathrm{~A}$ and the PRF of the bipolar current pulse is between $1 \mathrm{~Hz}$ and $10 \mathrm{kHz}$. The proposed inverter contains a high voltage source circuit for the rising edge and a Zener diodes circuit with constant voltage clamping for the falling edge. Measurement results are presented, which show the high quality triangular wave current pulse with linear rising edge and rapidly turnoff falling edge.
\end{abstract}

\section{Introduction}

Triangular wave current pulse can be used for mineral exploration, unexploded ordnance exploration, hydrogeological survey, engineering geological survey, geological mapping and so on[1]. It also has several merits against square wave in ground penetrating. It has the advantage that the primary field is simple and it is suitable for shallow low-resistivity target detection, as well as early signal acquisition and processing[2].

In this letter, a high voltage source circuit and inverter bridge circuit are designed to generate the bipolar triangular wave current pulse. For high voltage source circuit, five power modules are connected in series to provide the high power with stable DC voltage. For the inverter circuit, it has a full-bridge circuit of four MOSFETs, which are driven by four isolated PWM signals given by MCU. High and constant voltage clamping circuit is the essential part of this inverter to make the current pulse rise lineally to peak current and fall down rapidly to zero during the turn-off time. Finally, we can obtain periodic triangular wave current pulse with peak values more than $10 \mathrm{~A}$ and $\mathrm{PRF}$ of $1 \mathrm{~Hz}$ to $10 \mathrm{kHz}$ for different applications.

\section{Theoretical Analysis}

For a load such as small air-core coil, it is typically equal to a resistor and an inductor connected in series[3]. For a current pulse with linear edges, the corresponding voltage of the coil should be linear too. The reason will be described soon. A positive triangular wave current pulse is depicted in formula (1), and the negative cycle is the same to the positive one, except the polarity is different.

$$
i(t)= \begin{cases}\frac{I_{m}}{t_{r}} t, & 0 \leq t<t_{r} ; \\ I_{m}\left(1-\frac{t-t_{r}}{t_{f}}\right), & t_{r} \leq t<t_{r}+t_{f} ; \\ 0, & t_{r}+t_{f} \leq t<\frac{T}{2} .\end{cases}
$$

where $t_{r}$ is the rising time of the current pulse and $t_{f}$ is the falling time of the current pulse. $I_{m}$ is amplitude of the peak current. $T$ is the period of the current pulse. The corresponding equations of the coil is as following

$$
\left\{\begin{array}{l}
u_{L}(t)=L \frac{\mathrm{d} i(t)}{\mathrm{d} t} \\
u_{R}(t)=R i(t) \\
u(t)=u_{L}(t)+u_{R}(t)
\end{array}\right.
$$

where $u(t)$ is the voltage of the coil. $L$ and $R$ are the inductance and resistance of the load respectively. According to the formula (1) and the formula (2), the $u(t)$ is shown as formula (3)

$$
\mathrm{u}(\mathrm{t})= \begin{cases}\frac{L I_{m}}{t_{r}}+\frac{R I_{m}}{t_{r}} t, & 0 \leq t<t_{r} ; \\ \frac{L I_{m}}{t_{f}}+R I_{m}\left(1-\frac{t-t_{r}}{t_{f}}\right), & t_{r} \leq t<t_{r}+t_{f} ; \\ 0, & t_{r}+t_{f} \leq t<\frac{T}{2} .\end{cases}
$$

In practical application, it is difficult to generate this kind of voltage, so an approximate solution is applied. For a small air-core coil, it can be inferred that $L \mathrm{~d} i(t) / \mathrm{d} t \gg$ $R i(t)$, that is $u_{L}(t) \gg u_{R}(t)$, so $u(t)$ can be simplified to a constant voltage in formula (4) 


$$
u(t)= \begin{cases}\frac{L I_{m}}{t_{r}}, & 0 \leq t<t_{r} ; \\ \frac{L I_{m}}{t_{f}}+R I_{m}\left(1+\frac{t_{r}}{t_{f}}\right), & t_{r} \leq t<t_{r}+t_{f} ; \\ 0, & t_{r}+t_{f} \leq t<\frac{T}{2} .\end{cases}
$$

With a constant voltage, the exact formula of the current is showed below. The time constant $\tau$ of the coil can be get from $\tau=L / R, U_{z}$ is the constant clamping voltage of the falling edge.

rising edge

$$
i_{r}(t)=\frac{U}{R}\left(1-e^{-\frac{t}{\tau}}\right), 0 \leq t<t_{r}
$$

Falling edge

$$
i_{f}(t)=\left(I_{m}+\frac{U_{Z}}{R}\right) e^{-\frac{t-t_{r}}{\tau}}-\frac{U_{Z}}{R}, 0 \leq t<t_{r}
$$

The value of $t_{r}$ and $t_{f}$ is shown in formula (7)

$$
\mathrm{t}_{\mathrm{r}}=\tau \ln \left(\frac{U}{U-R I_{m}}\right), t_{f}=\tau \ln \left(1+\frac{R I_{m}}{U_{z}}\right)
$$

The derivative of the rising stage and the falling stage can be obtained by formula (8) and formula (9)

$$
\begin{gathered}
\frac{\mathrm{d} i_{r}(t)}{\mathrm{d} t}=\frac{U}{L} e^{-\frac{t}{\tau}}, 0 \leq t<t_{r} \\
\frac{\mathrm{d} i_{f}(t)}{\mathrm{d} t}=-\frac{1}{L}\left(R I_{m}+U_{Z}\right) e^{-\frac{t-t_{r}}{\tau}}, t_{r} \leq t<t+t_{f}
\end{gathered}
$$

In general, if $e^{-\frac{t}{\tau}}>0.95[4]$, that is $t / \tau<0.0513$, the $i_{r}(t)$ and $i_{f}(t)$ are deemed to be linear. The condition of the resistance of the coil can be simplified as (10)

$$
\ln \left(\frac{U}{U-R I_{m}}\right)<0.0513, \ln \left(1+\frac{R I_{m}}{U_{Z}}\right)<0.0513
$$

\section{Inverter Circuit}

From the analysis above, it is obviously that how to provide a high and constant voltage is the key point of the circuit. For the triangular wave current pulse, high voltage can be applied to make the current rise up and fall down linearly. Therefore, we apply active constant voltage[5] clamping to the rising edge and passive constant voltage clamping to the falling edge. The high voltage generation circuit for active constant voltage clamping is shown in Fig. 1.

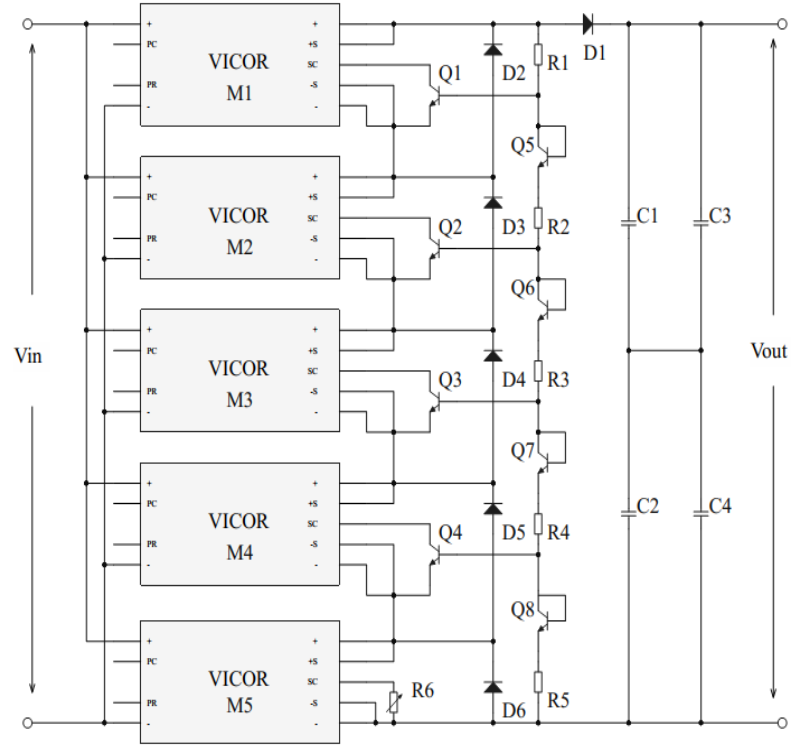

Fig. 1. Hight Voltage Generation Circuit.

VICRO module V24B48C250BL is chosen for the triangular wave pulse generation. It has input voltage of $24 \mathrm{~V}$, maximum output voltage of $48 \mathrm{~V}$. The power of this module is $250 \mathrm{~W}$ and the maximum current output is $7 \mathrm{~A} .5$ modules connected in series are needed to generate rising edge of the triangular wave. The total clamping voltage of the 5 modules is up to $240 \mathrm{~V}$. In this circuit, the diode D1 isolates the high voltage source and the inverter circuit. Diodes D2 to D6 protect each power module. Capacitors $\mathrm{C} 1$ to $\mathrm{C} 4$ are applied to store energy and increase the maximum current limit and their values are $220 \mu \mathrm{F}$.

For this application, the output voltage of the module is required to be adjusted. The configuration of Fig. 1 provides a reliable and inexpensive solution to realize voltage adjusted by R6 with a value of $10 \mathrm{k} \Omega$. In this solution, resistors R1 to R5 are all equal and their values are $10 \mathrm{k} \Omega$. All the transistors $\mathrm{Q} 1$ to $\mathrm{Q} 8$ are $2 \mathrm{~N} 222$. The effect of Q1 to Q8 is to adjust the voltage of each converter. When the output voltage of the converter M5 is adjusted, the voltages of convert M1 to M4 also change until they are all equal to the voltage of M5. Thus, the five converters have an effect on the total output voltage.

The full bridge circuit is shown in Fig. 2 and it has four MOSFETs[6]. The MOSFET module is SCT2280KE of ROHM Semicondctor. As mentioned above, a highvoltage passive clamping is used to the falling edge because the current must turn off as soon as possible to provide wide-band high frequencies for shallow TEM explorations.

When the power is supplied, QUL and QDR are turned on first, and the current passes D7, QUL, L, R and QDR to generate positive pulse[7]. Because the coil is inductive, the current rises in linear rule by the work of the high voltage circuit. When the current reaches the peak amplitude, QUL and QDR are turned off and QSW is turned on to let the current in the coil to go through the damping resistor RESn. At this time, the current has two ways to go. One way is to pass through the diode of QUR and QDL, diode D8 and Zener diodes circuit, the other one is to pass through QSW and RESn. The Zener diodes 
circuit form the high-voltage passive clamping circuit. The Zener diodes are 1N5369B which has Zener voltage of $51 \mathrm{~V}$, so the total clamping voltage of 10 diodes is about $510 \mathrm{~V}$. The capacitors C9 and C10 in parallel to the Zener diodes are used for protecting the Zener diodes from damage if the power is too large. The resistors R11 and $\mathrm{R} 12$ with value of $100 \mathrm{k} \Omega$ are used to discharge the contour of $\mathrm{C} 9$ and $\mathrm{C} 10$. The effect of the damping resistor RESn is to suppress current overshoot when the current decays to zero. What the value of RESn is selected depends on the coil.

Due to stray parameters[8] of the entire system, there is a high frequency oscillation at the early part of the turnoff current when the switch is suddenly cut off. The oscillation will affect the early electromagnetic data, so a RCD circuit parallel to each MOSFET is added to absorb and decrease the oscillation current[9].

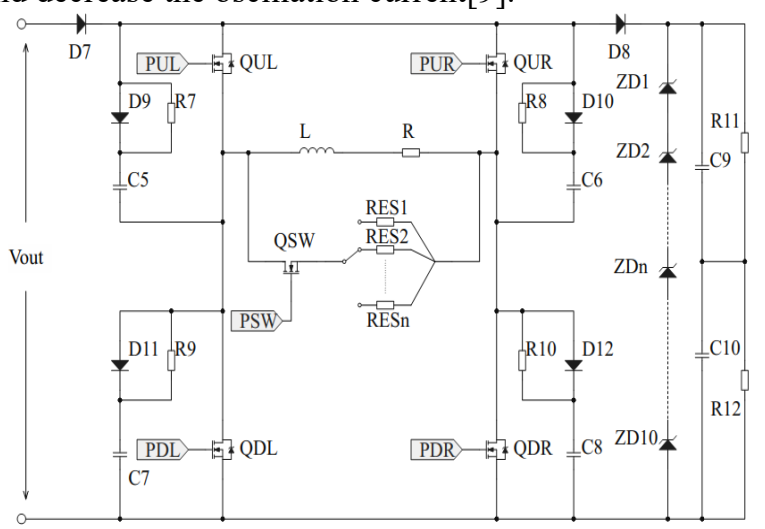

Fig. 2. Circuit Diagram.

\section{Measurement}

We use two different coils, the Coil A and Coil B to test the triangular wave current inverter. Both of the coils are $1 \mathrm{~m} \times 1 \mathrm{~m}$ square shape. The Coil A has 30 turns and the cross section of the wire is $2.5 \mathrm{~mm}^{2}$. It is equal to a $2 \Omega$ resistor connected in series with a $3 \mathrm{mH}$ inductor. The Coil B has 25 turns and the cross section of the wire is $4 \mathrm{~mm}^{2}$. It is equal to a $281 \mathrm{~m} \Omega$ resistor connected in series with a $1.65 \mathrm{mH}$ inductor. A high-accuracy current probe and high-voltage differential probe are used to measurement the current and the voltage waveforms of the coils.
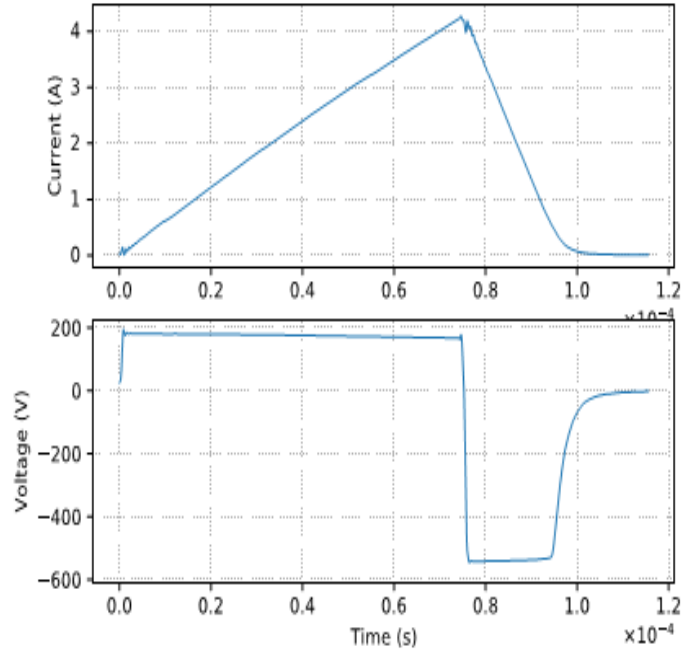

Fig. 3. Current and Voltage of Coil A.
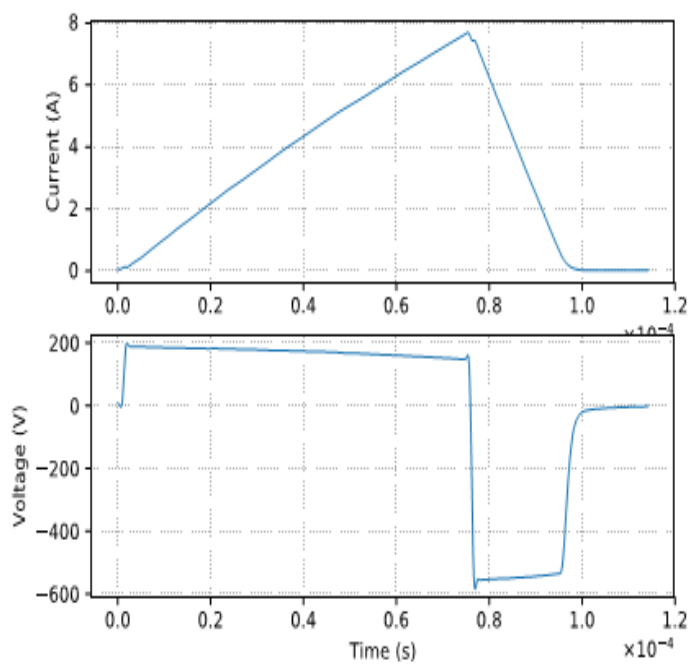

Fig. 4. Current and Voltage of Coil B.

The results of Coil A are shown in Fig. 3 and the results of Coil B is shown in Fig. 4. From Fig. 3 and Fig. 4, the current rises linearly to the peak in $75 \mu$ s with the constant clamping voltage of $200 \mathrm{~V}$. The current in Coil A reaches $4.3 \mathrm{~A}$ and decays to zero in $24.5 \mu \mathrm{s}$. The current in Coil B reaches $7.7 \mathrm{~A}$ and decays to zero in $22.6 \mu \mathrm{s}$. The turn-off current in both coils decay linearly and rapidly with the constant clamping voltage of $510 \mathrm{~V}$. Because they both have high linearity rising edge and falling edge, the quality of the triangular wave current is very good for applications.

However, there is a flaw of small oscillation in the current waveform due to the stray parameters and the oscillation of Coil B is smaller than Coil A. The designed RCD circuit could reduce but cannot completely remove the oscillation, so there will be a future work to suppress the oscillation and further enhance the quality of the current waveform.

\section{Conclusions}

The key point of the present circuit is how to generate triangular wave pulse current in practical application. At all, the circuit present above is able to produce a high 
quality triangular wave pulse current with linear rising edge and linear and rapid falling edge. The peak current is more than $10 \mathrm{~A}$ and its peak power is $1000 \mathrm{~W}$. The PRF of the pulse inverter is between $1 \mathrm{~Hz}$ and $10 \mathrm{kHz}$. It can completely meet the requirements of the practical application.

\section{Acknowledgements}

This work has been supported by Key Laboratory of Electromagnetic Radiation \& Sensing Technology, Chinese Academy of Sciences.

\section{References}

1. G. Hodges, T. Chen, Exploration Geophysics, 46, 5863(2014)

2. B. Nikls, H. Garbe, M. Schaarschmidt, International Symposium on Electromagnetic Compatibility - Emc Europe, 1-6(2017)

3. B. K. Sternberg, S. L. Dvorak, W. Feng, Journal of Applied Geophysics, 136, 211-218(2017)

4. K. Schoeman, P. Meyer, D. I. L. D..Villiers, Africon, 1-5(2013)

5. Y. Moursy, R. Iskander, M. M. Louërat, Mixed-Signal Testing Workshop, 1-6(2015)

6. B. Niklas, H. Garbe, M. Schaarschmidt, International Symposium on Electromagnetic Compatibility-Emc Europe. 1-6(2017)

7. H. S. Saini, J. M. Martinez, L. A. C. Lopes, Iet International Conference on Power Electronics, Machines and Drives IET, 1-6(2014)

8. J. A. Ferreira, Kane, I. J. Klinkert, IEEE Transactions on Industry Applications, 40, 213-219(2017)

9. M. Stork, Radioelektronika. IEEE, 1-4(2017) 